Association for Information Systems

AIS Electronic Library (AISeL)

Wirtschaftsinformatik 2021 Proceedings

Track 3: Student Track

\title{
Data Governance: State-of-the-Art
}

Giulio Behringer

Hochschule Heilbronn

Marcel Hizli

Hochschule Heilbronn

Follow this and additional works at: https://aisel.aisnet.org/wi2021

Behringer, Giulio and Hizli, Marcel, "Data Governance: State-of-the-Art" (2021). Wirtschaftsinformatik 2021 Proceedings. 1.

https://aisel.aisnet.org/wi2021/XStudent/Track03/1

This material is brought to you by the Wirtschaftsinformatik at AIS Electronic Library (AISeL). It has been accepted for inclusion in Wirtschaftsinformatik 2021 Proceedings by an authorized administrator of AIS Electronic Library (AISeL). For more information, please contact elibrary@aisnet.org. 


\title{
Data Governance: State-of-the-Art
}

\author{
Giulio Behringer ${ }^{1}$, Marcel Hizli ${ }^{1}$ \\ ${ }^{1}$ Hochschule Heilbronn, Fachbereich Wirtschaftsinformatik, M. Sc. Wirtschaftsinformatik - \\ Informationsmanagement und Data Science, Heilbronn, Germany \\ \{gbehring,mhizli\}@stud.hs-heilbronn.de
}

\begin{abstract}
Um heutzutage in der Wirtschaft bestehen zu können, langt es nicht mehr an alten Gewohnheiten und Unternehmensstrukturen festzuhalten. Vielmehr kommt es darauf an, im Zeitalter der Digitalisierung, Innovations- und Optimierungspotentiale rechtzeitig zu erkennen und stetig zu hinterfragen. Dies kann auf lange Sicht nur gewährleistet werden, indem eine geeignete Data Governance im Unternehmen etabliert wird. Neben der Data Governance spielt die IT-, Informations- und Corporate Governance eine wichtige Rolle, um organisatorische Rahmenbedingungen in Unternehmen zu installieren. In Bezug auf die oben beschriebene Ausgangslage soll mittels Literaturrecherche nach Fettke (2006), sowie Webster und Watson (2002) der aktuelle, wissenschaftliche Forschungsstand von Data Governance und die wechselseitigen Abhängigkeiten zu den angrenzenden Governancearten: Corporate-, Information-, und ITGovernance, dargestellt werden.
\end{abstract}

Keywords: Governance, Data Governance, Corporate Governance, ITGovernance, Information-Governance

\section{$1 \quad$ Einleitung}

Für ein besseres Verständnis wird zu Beginn der wissenschaftlichen Arbeit zuerst auf die Problem- und Fragestellung eingegangen, bevor die Zielsetzung Auskunft über den Mehrwert des wissenschaftlichen Beitrags gibt.

\subsection{Problemstellung}

Daten werden im Zeitalter der Digitalisierung als "das neue Öl" verkündet, jedoch kämpfen Organisationen immer noch damit, einen Mehrwert für ihr Unternehmen zu generieren [1]. Viele Organisationen sind an neuen Möglichkeiten im Kontext der Bewältigung von großen Datenmengen interessiert. Dennoch werden sie oft durch scheinbar langwierige Datenprobleme gehemmt [2]. Fragen der Qualität, Verfügbarkeit oder Genauigkeit erschweren die Bemühungen um Daten. Diese isoliert zu lösen, stellt jedoch nur eine kurzfristige Lösung dar [3]. Die Gewinnung von Nutzen aus Daten erfordert einen organisationsweiten Ansatz und kann als solche nicht von der IT allein gelöst werden. Hier wurde die Data Governance sowohl von Praktikern, als auch von 
Wissenschaftlern als vielversprechender Ansatz zur Lösung dieser organisatorischen Datenprobleme identifiziert [4, 5]. Dahingehend bietet eine genaue Abgrenzung, sowie die Interdependenzen angrenzender Governancedomänen Optimierungspotential, um einen wesentlichen Beitrag zur Wertschöpfung in Unternehmen beizusteuern [6, 7].

\subsection{Fragestellung}

Im Zeitalter der digitalen Transformation wird sich jedes Unternehmen die Frage stellen, welche Maßnahmen getroffen werden müssen, um eine optimale Nutzung der Daten und Information zu gewährleisten. Folglich muss eine Organisation zur Sicherstellung des regelkonformen Umgangs mit Daten eine Data Governance

etablieren. Hierbei gilt es die verschiedenen Bereiche des Corporate-, Information-, IT- und Data Governance genauer zu betrachten, um einen geeigneten Ordnungsrahmen für Unternehmen abstecken zu können.

Daraus abgeleitet, kristallisiert sich folgende Forschungsfrage heraus: Wie ist der aktuelle Stand der Wissenschaft bezüglich Data Governance?

Dahingehend kann die Forschungsfrage in weitere Teilfragen differenziert werden: Was ist Data Governance und welche wechselseitigen Beziehungen existieren bezüglich Corporate-, Information- und IT-Governance? Welche Herausforderung und Ziele stehen im Zusammenhang mit der Data Governance?

\section{$1.3 \quad$ Zielsetzung}

Das Ziel dieses wissenschaftlichen Beitrags ist es, einen Einblick in die Data Governance, sowie Corporate-, Informations-, und IT-Governance zu gewinnen. Mithilfe einer systematisch, wissenschaftlichen Literaturrecherche wird der aktuelle Wissensstand der Forschung auf dem Gebiet der Data Governance und angrenzenden Governacearten abgebildet. Auf Basis der gefundenen Literatur liegt der initiale Fokus auf der Charakterisierung, den Herausforderungen und Zielen von Data Governance, sowie einer vergleichenden Betrachtung von Ordnungsrahmen für die Corporate-, Informations-, IT- und Data Governance. Resultierend aus den Erkenntnissen des Beitrags sollen weitere Forschungsfelder aufgedeckt werden.

\subsection{Aufbau der Arbeit}

Die vorliegende wissenschaftliche Ausarbeitung teilt sich in insgesamt fünf Kapitel auf. In der Einleitung wird zunächst ein Überblick über den wissenschaftlichen Beitrag gegeben. Dabei wird zuerst die Problem- und Fragestellung erläutert, um daraufhin eine geeignete Zielsetzung zu formulieren. Damit die derzeitige Ausgangsbasis verständlich ist, werden im zweiten Kapitel die Grundlagen und die wichtigsten Begriffe erläutert, welche die Basis für die wissenschaftliche Ausarbeitung darstellen. Im nachfolgenden Kapitel 3 wird die Methodik zur Literaturanalyse dargelegt und genauer auf die Vorgehensweise eingegangen, wie die verwendete Literatur aus den gelisteten Datenbanken und Konferenzen herausgefiltert wurde. Das vorletzte der fünf Kapiteln präsentiert die Ergebnisse des Beitrags auf Basis der in Kapitel 3 beschriebenen 
Literatur und nimmt Bezug auf die eingangs gestellte Forschungsfrage. Im abschließenden Kapitel 5 findet die Zusammenfassung der Ergebnisse, sowie eine kritische Würdigung statt. Dies soll nochmals die gewonnenen Erkenntnisse verdeutlichen und einen Ausblick auf weitere Forschungsarbeiten liefern.

\section{Grundlagen und Begriffe}

Im folgenden Abschnitt werden die relevanten Grundlagen und Begriffe näher erläutert. Für eine optimale Ausgangslage wird zuerst die Thematik der Data Governance erläutert. Im Anschluss daran werden die wichtigsten, angrenzenden GovernanceTypen: Corporate-, IT-, und Information-Governance näher beschrieben, um eine gemeinsame Basis zur Beantwortung der Forschungsfrage zu schaffen.

Für ein kollektives Verständnis der verschiedenen Governancearten wird der Begriff „Governance“ vorab nach [8] definiert: Governance „bezeichnet die verantwortungsvolle, nachhaltige und auf langfristige Wertschöpfung gerichtete Organisation und Steuerung von Aktivitäten und damit das gesamte System interner und externer Leistungs-, Kontroll- und Überwachungsmechanismen.“.

\subsection{Data Governance}

Data Governance ist eine Disziplin des Data Managements zur Verwaltung von Datenund Informationsbeständen in einer Organisation. Dies erfolgt durch eine formelle Aufsicht über die Personen, Prozesse, Technologien und Geschäftszweige, die Datenund Informationsergebnisse beeinflussen, um die Geschäftsleistung zu steigern. Mit Hilfe von Standards und Richtlinien sorgt Data Governance dafür, dass die Qualität, sowie der Schutz und die Sicherheit von Daten gewährleistet und die rechtlichen Rahmenbedingungen eingehalten werden [4]. Laut [9] kann Data Governance als ein organisatorischer Ansatz zur Datenverwaltung beschrieben werden, der eine Reihe von Richtlinien und Verfahren formalisiert, um den gesamten Lebenszyklus von Daten abzudecken.

Im Vordergrund von Data Governance stehen Richtlinien und Vorgaben für die korrekte Handhabung und Pflege von Daten. Dabei orientiert sich die Data Governance stark an der betriebswirtschaftlichen Zielsetzung der Organisation [10]. Dabei hilft Data Governance Unternehmen, eine Reihe von Regeln und Vorschriften für die Verwaltung von Daten zu verabschieden, um die Standards oder Anforderungen der Organisation zu erfüllen [11].

\subsection{Corporate Governance}

Die Corporate Governance stellt einen institutionellen Ordnungsrahmen für Organisationen dar. Daraus abgeleitet lassen sich konkrete Vorgaben und Leitlinien definieren, die wiederrum auf sämtliche Bereiche der Organisation adaptierbar sind [10]. Ein wichtiger Bestandteil des Corporate Governance tangiert wesentliche Bereiche der Unternehmensführung hinsichtlich Kunden, Prozesse, Richtlinien, 
Gesetze und Institutionen. Zudem spielt der Umgang und die Zusammenarbeit mit verschiedene Interessensgruppen, wie Direktoren, Manager, Aktionäre, Mitarbeiter, Lieferanten, Kunden, etc. eine wichtige Rolle, um Unternehmensziele zu erreichen. Die Corporate Governance wird anhand verschiedener Modelle im Unternehmen umgesetzt. Idealerweise sollte die Corporate Governance eine produktive und effiziente Arbeitsweise ermöglichen und gleichzeitig Korruption und Machtmissbrauch verhindern [12].

\subsection{IT-Governance}

[13] beschreiben die IT-Governance als ein Framework, das Unternehmen bei der Entscheidungsfindung, Bewertung und Implementierung im Kontext von IT unterstützt. IT-Governance ist eine Art von Institution, welche diverse Managementprinzipien aufführt, um den Richtlinien seitens Business und der IT zu entsprechen. Die Definition von [12] legt den Fokus auf Prozesse und Strukturen und lautet wie folgt: „IT-Governance ist der Prozess und die Struktur, die sicherstellen, dass Unternehmen ihre IT-Investitionen angemessen einsetzen, um sicherzustellen, dass die daraus resultierenden Aktivitäten - ob Programme, Projekte oder Operationen, die sie finanzieren - ordnungsgemäß ausgeführt werden und die gewünschten Ergebnisse erzielen“. Dabei liegt der Fokus auf den Führungs- und Organisationsstrukturen, sowie Prozessen, die sicherstellen, dass die IT die Strategie und die Ziele der Organisation aufrechterhält und erweitert [14].

\subsection{Information Governance}

Die Information Governance beschreibt die Art und Weise, wie in einer Organisation inklusiver aller Geschäftsprozesse Daten und Informationen verwaltet werden [15]. [16] definieren die Information Governance als einen zeitgemäßen Ansatz, um die Nutzung von Informationen besser zu verwalten und ihren Wert innerhalb und außerhalb von Organisationen zu schützen und zu maximieren. In Übereinstimmung mit [16] umfasst die Information Governance ein Umfeld von Möglichkeiten, Regeln und Entscheidungen für die Bewertung, Erstellung, Sammlung, Analyse, Verteilung, Speicherung, Nutzung und Kontrolle von Informationen [16, 17]. Dabei ist die Information Governance keine einmalige Angelegenheit, sondern ein kontinuierlicher Prozess. Die Information Governance legt zum einen die Entscheidungsrechte fest und stellt zugleich einen Rahmen für die Rechenschaftspflicht, um ein wünschenswertes Verhalten bezüglich der Bewertung, Erstellung, Speicherung, Nutzung, sowie Archivierung und Löschung von Informationen und Daten zu fördern. Dies umfasst neben den Prozessen und Rollen, auch Standards und Metriken, die eine optimale Nutzung der Informationen in Organisationen sicherstellen sollen [18]. 


\section{Methodik zur Literaturanalyse}

In diesem Abschnitt wird die Methodik zur Literaturanalyse detailliert dargestellt. Dabei wird das Verfahren näher erläutert, mit welcher Vorgehensweise die Literaturanalyse durchgeführt wurde. Daraufhin wird näher auf die verwendeten Literaturdatenbanken und Konferenzen eingegangen, sowie die verwendeten Suchabfragen und Suchtherme. Zur besseren Nachvollziehbarkeit der Literaturanalyse werden noch die verwendeten Suchfilter, sowie die Eingrenzung des Zeitrahmens der Literatursuche und abschließend die Charakterisierung der Literaturanalyse angegeben.

\subsection{Verfahren}

Für einen ersten Einblick in das Themengebiet Data Governance, sowie die bereits genannten angrenzenden Themenfelder, wird eine Literaturanalyse der deutsch- und englischsprachigen Literatur durchgeführt. Mit Hilfe einer strukturierten Literaturanalyse nach [19] ist das Ziel möglichst viel relevante Literatur aufzuzeigen. Die anschließende Vorwärts- und Rückwärtsrecherche nach [20] dient zur Überprüfung der Vollständigkeit der Literatur in Bezug zur Forschungsfrage.

\subsection{Literaturquellen}

Einen genaueren Überblick über den Quellenfindungs- und Auswertungsprozess zeigt die nachstehende Tabelle.

Tabelle 1: Auswertung der Literaturrecherche

\begin{tabular}{lllll}
\hline Quelle & Term 1 & Term 2 & Term 3 & Term 4 \\
\hline AISel & 275 & 67 & 41 & 29 \\
ACM & 263 & 22 & 14 & 7 \\
Emerald & 170 & 26 & 23 & 28 \\
IEEE & 114 & 5 & 6 & 3 \\
SL & 77 & 80 & 76 & 66 \\
AMCIS & 51 & 13 & 8 & 5 \\
ECIS & 39 & 10 & 4 & 2 \\
\hline Abfrageergebnisse & $\mathbf{9 8 9}$ & $\mathbf{2 2 3}$ & $\mathbf{1 7 2}$ & $\mathbf{1 4 0}$ \\
\hline Duplikate & 723 & 150 & 121 & 113 \\
Sichtung der Abstracts & 117 & 44 & 37 & 23 \\
Wissenschaftliche & 46 & 31 & 24 & 19 \\
Eignung & & & & \\
Inhaltliche Relevanz & 6 & 7 & 2 & 3 \\
\hline Rück- und Vorwärtssuche & & & \multicolumn{2}{c}{30} \\
\hline Summe der Beiträge & & & & \\
\hline
\end{tabular}

Die relevante Literatur konnte mittels folgender Literaturdatenbanken: AIS Electronic Library (AISel), Association for Computing Machinery Digital Library (ACM), 
Institute of Electrical and Electronics Engineers (IEEE), Emerald Insights, und Springer Link ermittelt werden. Des Weiteren wurde die Americas Conference on Information Systems (AMCIS) und die European Conference on Information Systems (ECIS) auf potenzielle Literatur überprüft.

Um möglichst effizient bei der Literaturrecherche vorzugehen, eignet es sich spezielle Suchbegriffe und Suchabfragen für die jeweiligen Datenbanken zu verwenden. Für einen ersten Überblick zur Thematik der Data Governance zu bekommen, wurde der Suchterm: (Suchterm 1: „Data Governance“) verwendet. Auf dieser Basis wurden die angrenzenden Governance-Typen: Corporate-, Information- und IT-Governance genauer untersucht, um versteckte Potential für die Data Governance Implementierung zu identifizieren. Für diese tiefergehende Analyse wurden insgesammt drei Suchterme verwendet: (Suchterm 2: ,, "Data Governance" AND ("IT-Governance" OR "IT Governance"), (Suchterm 3: ,, "Data Governance" AND ("Informationsgovernance" OR "Information Governance"), (Suchterm 4: ,, "Data Governance" AND ("Corporate Governance").

Hierbei wurde für die Springer Link Datenbank der Suchfilter „Computer Science“ genutzt. Für die restlichen aufgeführten Datenbanken wurden keine weiteren Filtermöglichkeit verwendet. Ein wichtiger Eingrenzungsfaktor für die Literaturrecherche ist der Zeitraum. Allerdings wurde auf die Eingrenzung des Zeitrahmens bewusst verzichtet, da es keine Notwendigkeit gibt, den zeitlichen Rahmen im Kontext der Data Governance zu beschränken.

Auf Grundlage dieser Literaturbasis wurde eine Selektion anhand der Kriterien Duplikate, Sichtung der Abstracts, wissenschaftliche Eignung und inhaltliche Relevanz durchgeführt. Anschließend konnten mittel Vorwärts- und Rückwärtsrecherche nach Webster und Watson (2002) weitere 30 Quellen aufgefunden werden.

\section{$4 \quad$ Ergebnisse}

Die Basis für die nachstehende Begutachtung der Literaturanalyse wurde bereits in Kapitel 3 geschaffen. Zur Beantwortung der Forschungsfrage wird in diesem Abschnitt die recherchierte Literatur ausgewertet und weitere Hypothesen für eine expliziert, zukünftige Forschung aufgestellt.

\subsection{Charakteristik der Data Governance}

Allgemeinbetrachtet liegt der Fokus der Data Governance auf Daten und Informationen. Hierbei können Daten als „Rohmaterial“" klassifiziert werden, währenddessen Informationen als „Daten im Kontext“ bezeichnet werden [21]. Wang und Otto verfolgen ähnliche Ansätze hinsichtlich Daten und Qualität. Wang betrachtet die Datenqualität als die Gebräuchlichkeit von Daten, während Otto die Aussage präzisiert und feststellt, dass Daten nur dann einen Wert haben, sofern sie verwendet werden [4, 22]. Vor diesem Hintergrund zielt Data Governance darauf ab, den Wert von Datenbeständen in Organisationen zu maximieren, was zugleich die Wichtigkeit des Data Quality Managements hervorhebt [4]. Wende verweist auf eine klare 
Abgrenzung der beiden Begriffe. Data Governance ergänzt das Data Management, aber kann dieses nicht ersetzen [23]. Im Folgenden wird die Charakteristik anhand der gestaltungsorientierten Ansätze der Rollenverteilung, Aufgaben und Zuständigkeiten näher beschrieben. Auf organisatorische Aspekte, wie z.B. Strategie, Finanzierung, Metadatenmanagement, Datenstandards, Richtlinien und Kennzahlen wird nicht weiter eingegangen [24].

Personelle Strukturen und Verantwortlichkeiten im Data Governance

Ein wichtiger Bereich der Data Governance stellt die Rollenverteilung bzw. die Verteilung der Verantwortlichkeiten von Aufgaben dar. Das nachstehende Schaubild verdeutlicht die Rollenverteilung innerhalb des Data Quality Managements.

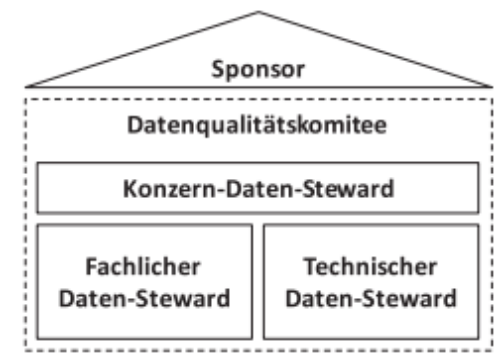

Abbildung 1: Rollenverteilung nach [10]

Hierbei muss die Unternehmensleitung die Data Governance-Richtlinien vorleben und absegnen. Die Rolle des Sponsors steht für die strategische Ausrichtung des Datenqualitätsmanagement und stellt die Unterstützung durch die Geschäftsleitung sicher [10, 23]. Das Datenqualitätskomitee fungiert als Bindeglied zwischen strategischer- und operativer Ebene und sorgt für die Festlegung von Richtlinien und Standards. Im Rahmen des Datenqualitätskomitee werden verschiedene Projekte zur Datenqualitätsverbesserung unter der Leitung des Konzern-Daten-Stewards mit Hilfe von fachlichen- und technischen Daten-Stewards durchgeführt. Der Konzern-DatenSteward ist hauptsächlich für die Umsetzung und Überwachung der Beschlüsse und Entscheidungen zuständig. Fachliche Daten Stewards hingegen sind Mitarbeiter der einzelnen Fachbereiche und sind für die unternehmensweiten Datenstandards verantwortlich. Der technische Daten-Steward beschäftigt sich mit allen Fragestellungen rund um das Thema Datenarchitektur und Systemunterstützung für das Datenqualitätsmanagement [10].

[25] nennen weitere, essenzielle Rollen im Zuge der Data Governance. Data Custodian müssen eingesetzt werden, um die Qualität von Bestandsdaten zu gewährleisten und Fragen hinsichtlich der Datenqualität zu beantworten. Zudem fungieren sie als Schnittstelle zwischen verschiedenen Personengruppen, um je nach Bedarf mit dem Data Governance Rat zu kommunizieren. Des Weiteren können Data Custodians als Führungskraft für Daten-Stewards betrachtet werden. Abgerundet werden die Rollen innerhalb der Data Governance mit Benutzergruppen, die von Data Stewards geleitet werden, bestehend aus Personen von verschiedensten Abteilungen, 
welche sich mit Daten oder deren dazugehörigen Infrastruktur beschäftigen. Neben den Daten Stewards führen die Autoren [26] und [27] die Prozessverantwortlichen, das Data Governance Office und das Data Governance Board auf, um eine Data Governance mit den nötigen Rollen innerhalb des Unternehmens zu etablieren. [5] greifen die erwähnten personellen Strukturen auf und ordnen sie entsprechenden Domänen im Data Governance ein. Diese werden anhand der Domänen Datenprinzipien, Datenqualität, Metadaten, Datenzugänglichkeit und Datenlebenszyklus kategorisiert. Weiterführende Rollen, wie zum Beispiel Datenqualitätsmanager, Datenqualitätsanalyst, Datenarchitekt oder der Datensicherheitsoffizier können daraus abgeleitet werden.

[28] greift die bereits erwähnten Rollen auf und spiegelt diese bezüglich der Datenqualität in einem Data Ownership Konzept wider. Dieses Konzept ist als ein Gestaltungsrahmen für Teilbereiche von Unternehmensdaten und den Verantwortlichen für deren Qualität und Verfügbarkeit zu betrachten. Der Vorteil dieses Konzeptes ist die klare Abgrenzung der Verantwortlichkeiten, um positive Maßnahmen in Hinsicht der Datenqualität einzuleiten. Innerhalb des Konzeptes existieren drei idealtypische Rollenverteilungen. Zum einen ist der Datenerfasser verantwortlich für die Extraktion, Verarbeitung und Speicherung von Daten, zum anderen legt der Datennutzer Anforderungen fest und verarbeitet die weitergeleiteten Daten. Der Datenverarbeiter hingegen ist für die Modifizierung und Weitergabe von Daten anhand der Unternehmensprozesse zuständig.

Zudem existieren nach [28] weitere Rollen im Zuge der Data Governance. Die DataOwner sind die Verantwortlichen, um Daten qualitativ hochwertig bereitzustellen, währenddessen die Datenprüfer die Ergebnisse überwachen und Risiken identifizieren. Daraus können entsprechende Gegenmaßnahmen eingeleitet werden, welche zudem von Data Riskmanagern unterstützt werden, damit Standards, Richtlinien und Normen eingehalten werden, um anhand dessen Datenprozesse auf neue mögliche Risiken zu evaluieren.

\section{Tätigkeitsfelder der Data Governance}

Der Verantwortungsbereich der Data Governance teilt sich zwischen dem Management und allen Mitarbeiter der Organisation auf. Dagegen liegt die Datenqualität in der Verantwortung aller und trägt maßgeblich zur Leistung und Erfolg des Unternehmens bei [27]. Um die möglichen Bereiche der Date Governance eingliedern zu können, ermittelte Weber diverse Betrachtungsgegenstände, die von der Data Governance aufgenommen und behandelt werden. Hierzu zählen das Datenqualitätsmanagement, das Stammdatenmanagement, das Informationsmanagement und die Business Intelligence [24].

Nach [4] beschäftigt sich Data Governance mit den folgenden Fragen: "Welche Entscheidungen im Kontext von Daten müssen im Unternehmen getroffen werden?", "Welche Rollen existieren im Rahmen des Entscheidungsprozesses?", "Inwiefern werden diese Rollen in den Entscheidungen beteiligt?". Des Weiteren befasst sich die Data Governance mit den Hauptaufgaben bezüglich des Managements von Datenqualität, der Datenarchitektur, des Datenschutzes, der Metadaten und des Informationslebenszyklus. Zudem behandelt die Data Governance die Dokumentation 
von rechtlichen Komponenten in Bezug auf Daten [4]. Anhand einer Umfrage von [29] mit 190 Unternehmen konnten die Autoren anhand einer qualitativen Analyse folgende Aufgaben im Bereich der Data Governance ermitteln. Basierend auf der Umfrage sind die Kernaufgaben der Data Governance das unternehmensweite Standardisieren von Definitionen in Bezug auf Daten, sowie das Festlegen von Standards, Richtlinien, Prozessen und Strategien bezüglich Informationen. Zudem zählt das Unterstützen von Abteilungen im Bereich Data Warehouse und Business Intelligence, das allgemeine Definieren und Festlegen von Regeln, und das Selektieren und Betreuen von Projekten hinsichtlich Datenqualität zu den Hauptaufgaben der Data Governance.

\section{Zuständigkeiten}

Innerhalb von Zuständigkeiten werden Verknüpfungen zwischen den ermittelten Rollen und Aufgaben gezogen. Inwiefern die Zuständigkeiten der jeweiligen Rollen im Zusammenhang von Aufgaben gestaltet werden, ist unternehmens- und kontextabhängig [10]. Rollen und die dazugehörigen Zuständigkeiten sind unerlässlich. Dadurch wird gewährleistet, dass ein klares Management und ein Verantwortlichkeitsgefühl der Daten entstehen. Sofern Personen verantwortlich gemacht werden können, kann davon ausgegangen werden, dass weniger Fehler in das System gelangen, und dass Fehler früher erkannt und gelöst werden, was wiederum zu effizienteren Prozessen führt [30].

Dies greift [23] weiter auf und erstellte hierfür eine Konzeptmatrix, welche in der folgenden Abbildung zu erkennen ist. Während innerhalb von operativen Tätigkeiten der technische Data Steward zuständig ist, liegt der Zuständigkeitsbereich bei strategischen Aufgaben beim Data Governance Rat oder beim Chief Steward. Der Business Data Steward unterstützt in verschiedensten Aufgaben. Die Rolle des Sponsors steht für die strategische Ausrichtung und stellt die Unterstützung durch die Geschäftsleitung sicher [10, 23].

\begin{tabular}{|c|c|c|c|c|c|c|}
\hline $\begin{array}{l}\text { Roles } \\
\text { Decision Areas }\end{array}$ & $\begin{array}{l}\text { Executive } \\
\text { Sponsor }\end{array}$ & $\begin{array}{c}\text { Data } \\
\text { Governance } \\
\text { Council }\end{array}$ & $\begin{array}{l}\text { Chief } \\
\text { Steward }\end{array}$ & $\begin{array}{c}\text { Business } \\
\text { Data } \\
\text { Steward }\end{array}$ & $\begin{array}{c}\text { Technical } \\
\text { Data } \\
\text { Steward } \\
\end{array}$ & $\ldots$ \\
\hline $\begin{array}{l}\text { Plan data quality } \\
\text { initiatives }\end{array}$ & A & $\mathbf{R}$ & C & I & $\mathbf{I}$ & \\
\hline $\begin{array}{l}\text { Establish a data } \\
\text { quality review } \\
\text { process }\end{array}$ & I & A & $\mathbf{R}$ & C & C & \\
\hline $\begin{array}{l}\text { Define data producing } \\
\text { processes }\end{array}$ & & A & $\mathbf{R}$ & C & C & \\
\hline $\begin{array}{l}\text { Define roles and } \\
\text { responsibilities }\end{array}$ & A & $\mathbf{R}$ & C & I & $\mathbf{I}$ & \\
\hline $\begin{array}{l}\text { Establish policies, } \\
\text { procedures and } \\
\text { standards for data } \\
\text { quality }\end{array}$ & A & $\mathbf{R}$ & $\mathbf{R}$ & C & C & \\
\hline $\begin{array}{l}\text { Create a business } \\
\text { data dictionary }\end{array}$ & & A & C & C & $\mathbf{R}$ & \\
\hline $\begin{array}{l}\text { Define information } \\
\text { systems support }\end{array}$ & & I & $\mathbf{A}$ & C & $\mathbf{R}$ & \\
\hline$\cdots$ & & & & & & \\
\hline
\end{tabular}

Abbildung 2: Konzeptmatrix der Zuständigkeiten nach [23] 
Die Autoren [31] greifen die Verteilung der Zuständigkeiten auf und beziehen diese stattdessen auf die Tätigkeitsbereiche der Datenqualitätsstrategie, der Datenpflegeprozesse, sowie den Datenqualitätszielgrößen oder dem Helpdesk. Zudem wird die Rolle „Shared Service Center" eingeführt, welcher überwiegend für die genannten Aufgaben verantwortlich ist. In diesem Kontext wird ein Data Quality Board zur Überwachung verwendet, wobei der technische Data Steward im Gegensatz zum Modell zuvor überwiegend informiert wird.

\subsection{Ziele und Herausforderungen der Data Governance}

Mit Hilfe der Data Governance soll in Organisationen eine effektivere Entscheidungsfindung ermöglicht und operative Reibungsverluste verringert werden. Zudem zielt das Data Governance darauf ab, die Interessen, Werte und Rechte der Stakeholder zu wahren [32]. Des Weiteren sollten in den Zielen der Data Governance Schulungsmöglichkeiten für Management und Personal fest verankert sein, um gemeinsame Ansätze für Datenprobleme anzunehmen, standardisierte, wiederholbare Prozesse aufzubauen, Kosten zu senken und die Effektivität durch Bündelung von Synergieeffekten zu erhöhen und die Transparenz der Prozesse sicherzustellen [33-35]. Zur Erreichung der Ziele muss im Rahmen der Data Governance einige Herausforderungen gemeistert werden, wie z.B. die Datenqualität. Hierbei spielen die Dimensionen der Datenqualität, wie Genauigkeit, Aktualität, und Konsistenz hinsichtlich der Bewertung eine bedeutende Rolle. Zudem sollten die Dimensionen durch Aktivitäten verbessert werden, die direkt auf Daten oder Prozesse bezüglich des Datenaustauschs und der Datenaufbereitung einwirken [36]. Eine weitere Herausforderung stellt die Daten-Modellierung, sowie die Daten- und SchemaIntegration dar. Hinsichtlich der Modellierung liegt der Fokus auf der Repräsentation von Datenklassen in Form eines konzeptuellen Modells, d.h. eines Modells, dessen linguistische Kategorien die Aspekte hervorheben, die mit der Bedeutung von Daten zusammenhängen. Für die Integration von Daten müssen Technologien bereitstehen, die es ermöglichen, verschiedene unabhängige Datenbanken abzufragen und auf sie zuzugreifen. Die Schema-Integration stellt eine Hürde dar bezüglich des Prozesses der Harmonisierung konzeptioneller Beschreibungen von Daten über heterogene Datenbanken hinweg. Eine weitere Herausforderung betrifft das Data Governance Management. Hierbei richtet sich die Betrachtung auf die Gesamtheit der Verantwortlichkeiten und Aktivitäten und ihre Anordnung in der Organisation, die es ermöglichen, die Qualität und das Integrationsniveau der Daten zu verwalten, zu überwachen, zu steuern und zu verbessern [37]. Des Weiteren wird die Unterstützung des Top Managements und des Change-Managements benötigt, um die Ziele der Data Governance realisieren zu können. Abhilfe kann hierbei ein unternehmenseinheitlicher Ansatz, sowie eine unternehmensweite Prozessharmonisierung schaffen [24].

\subsection{Interdependenzen zu angrenzenden Governancegebieten}

Mit dem Aufkommen neuer Governancearten und -gebieten steigt der Bedarf an klaren Abgrenzungen und Definitionen, um Missverständnisse zu vermeiden. Neben der 
Corporate Governance, Information Governance, IT-Governance und Data Governance, gehört in jüngster Zeit die Cloud Computing Governance zu einer weiteren Domäne. Auf Letztgenanntes wird im nachfolgenden Beitrag nicht näher eingegangen. Es ist daher wichtig, zwischen diesen Domänen zu unterscheiden und zu definieren, wie sie miteinander verbunden sind, insbesondere im Hinblick auf die Data Governance. Abbildung 3 verdeutlicht die wechselseitigen Abhängigkeiten der verschiedene Governancedomänen [32].

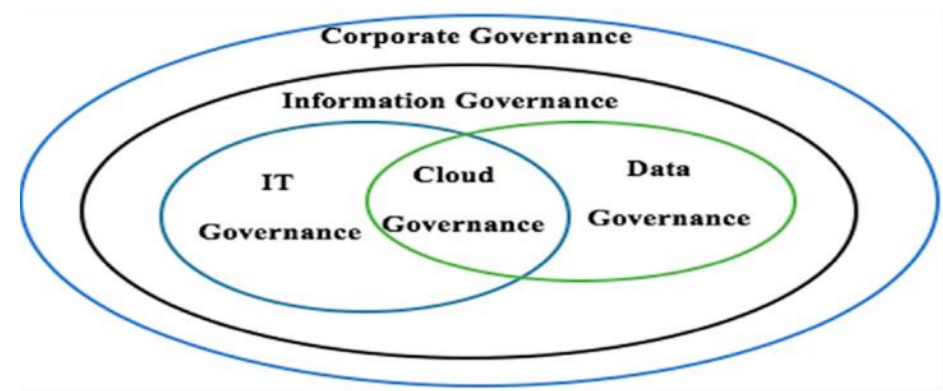

Abbildung 3: Die Interdependenzen der Governance-Domänen [32]

Der Begriff Governance bezieht sich im Allgemeinen auf die Art und Weise, wie eine Organisation sicherstellt, dass Strategien festgelegt, überwacht und erreicht werden [38]. Corporate Governance gibt die Richtlinien anhand von Verknüpfungsketten für die angrenzenden Governancetypen vor. Dahingehend beeinflusst die Corporate Governance im Wesentlichen die IT-Governance, die wiederum Einflüsse auf die Data Governance hat [39]. Zudem bestätigen [12] , dass Corporate Governance einen starken Einfluss auf die IT-Governance hinsichtlich einer zentralisierten oder dezentralen Ausrichtung aufweist. Des weiteren argumentieren [23] und [40] in gleicher Weise, dass Data Governance und IT-Governance die Prinzipien der Corporate Governance befolgen.

Hierbei entwickelt und implementiert die Data Governance unternehmensweite Datenpolitik, Richtlinien und Standards, die mit der Mission, Strategie, Werte, Normen und Kultur der Organisation übereinstimmen und in der Corporate Governance verankert sind [32]. Neben Größe und Wettbewerbsstrategie, zählt Corporate Governance zu den wichtigsten Einflussfaktoren der Data Governance [10]. Da die IT zum Rückgrat jeder Organisation geworden ist, ist die IT-Governance integraler Bestandteil jeder Geschäftsstrategie und fällt unter der Corporate Governance. Die Komplexität und das Volumen der Daten wächst, auf Grund von datengetriebenen Unternehmensentscheidungen und -prozessen, im gleichen Maße, wie der Bedarf an klaren Richtlinien und Vorgaben der Data Governance [41, 42]. Versuche, Daten zu verwalten, scheiterten zuvor, da sie von der IT gesteuert und von starren Prozessen und fragmentierten Aktivitäten beeinflusst wurden [43]. Allerdings existieren auch essenzielle Unterschiede zwischen Data- und IT-Governance [23, 44]. Während die Data Governance für die Verwaltung von Datenbeständen konzipiert wird, trifft die IT- 
Governance Entscheidungen über IT-Investitionen, das IT-Anwendungsportfolio und das IT-Projektportfolio. In der Praxis ist die IT-Governance in erster Linie auf die Hardware und Anwendungen eines Unternehmens ausgerichtet, nicht auf seine Daten. Die Information Governance scheint eine Lücke zu füllen, die von den bestehenden Governancestrukturen noch immer nicht ausgefüllt wurde [45]. Der Kerngedanke der Information Governance zielt auf die Minimierung von Problemen bezüglich der Informationsasymmetrie, indem Verknüpfungspunkte zu IT-Governance gebildet werden [46]. [38] greifen den Gedanken auf, und weisen auf die Verwendung von ITGovernance Mechanismen in der Information Governance hin. [47] führen fort, dass wesentliche Praktiken der IT-Governance für die Information Governance berücksichtigt werden können im Hinblick, wie Information geregelt werden. Des Weiteren ist die Data Governance ein Bestandteil der Information Governance. Während Information Governance alle Arten von unstrukturierten und strukturierten Informationen betrifft, hat Data Governance einen begrenzteren Anwendungsbereich, der sich nur auf strukturierte Daten konzentriert [48].

\section{$5 \quad$ Fazit und Ausblick}

In diesem wissenschaftlichen Beitrag, aufbauend auf einer klaren Abgrenzung der Fachbegriffe Data Governane, sowie die angrenzenden Governancetypen: Corporate-, Information, und IT- Governance, konnten mittels Literaturrecherche nach [19], und einer Vorwärts- / Rückwärtsrecherche nach [20], wichtige Beiträge über den aktuellen, wissenschaftlichen Forschungsstand zur Thematik von Data Governance ermittelt werden. Auf dieser Grundlage konnte durch eine ausführliche Literaturanalyse wichtige Erkenntnisse gewonnen werden. Die Charakteristika sorgt für ein besseres Verständnis zur Thematik von Data Governance. Die Rollenverteilung, sowie Aufgaben und Zuständigkeiten sind dahingehend elementare Bestandteile einer erfolgreichen Data Governance. Zudem weist die Data Governance starke Berührungspunkte zu Data Quality Management auf.

Des Weiteren konnten wichtige Herausforderungen und Ziele identifiziert werden. Die Auswertung der Literaturrecherche zeigte, dass keine klare Abgrenzung der einzelnen Governancedomänen existiert. Vereinzelte Beiträge konnten die Abhängigkeiten und Schnittstellen einzelner Governantypen darstellen. Daraus abgeleitet wurde ersichtlich, dass keine allgemeingültige Meinung zur Einordnung der Interdependenzen der verschiedenen Governanceytpen vorliegt. Die Corporate Governance beeinflusst im Wesentlichen die angrenzenden Governancearten, speziell die IT-Governance. Zudem konnte festgestellt werden, dass die Information-, IT- und Data-Governance untereinander Querbezüge aufweisen. Mit diesem Beitrag konnte dahingehend die Interdependenzen der Corporate-, Information-, IT- und DataGovernance aufgezeigt und dargestellt werden.

Resultierend aus den Erkenntnissen des Beitrags konnten neue Forschungsfelder aufgedeckt werden. Auf der einen Seite wurden weitere Governancearten, wie z.B. Cloud-Governance, oder Security-Governance identifiziert, allerdings nicht näher erläutert. Auf der anderen Seite bieten Einfluss-, Gestaltungs- und Erfolgsfaktoren der 
Data Governance weitere interessante Ansätze für eine zukünftige Forschungsarbeit, da auf Grundlage der Literaturrecherche nur vereinzelte Beiträge ermittelt werden konnten und dahingehend keine tiefergehende Betrachtung erfolgte. Für zukünftige Forschungsarbeiten bietet es sich an, folgenden Hypothesen nachzugehen:

- Welche wechselseitigen Abhängigkeiten existieren im Hinblick auf Cloud- und Security- Governance im Kontext von Corporate-, Information-, IT- und DataGovernance?

- Welche Einfluss-, Gestaltungs-, und Erfolgsfaktoren sind für das Data Governance ausschlaggebend?

- Welche Modelle und Frameworks existieren zur Umsetzung und Implementierung von Data Governance?

\section{References}

1. Ransbotham, S., Kiron, D., Prentice, P.: Beyond the hype: The hard work behind analytics success (2016)

2. Thompson, N., Ravindran, R., Nicosia, S.: Government data does not mean data governance: Lessons learned from a public sector application audit. Government Information Quarterly 32, 316-322 (2015)

3. Brous, P., Janssen, M., Vilminko-Heikkinen, R.: Coordinating Decision-Making in Data Management Activities: A Systematic Review of Data Governance Principles. In: Scholl, H.J., Glassey, O., Janssen, M., Klievink, B., Lindgren, I., Parycek, P. (eds.) Electronic government. 15th IFIP WG 8.5 international conference, EGOV 2016, Guimarães, Portugal, September 5-8, 2016 ; proceedings, pp. 115-125. Springer, Switzerland (2016)

4. Otto, B.: Data Governance. WIRTSCHAFTSINFORMATIK 53, 235-238 (2011)

5. Khatri, V., Brown, C.V.: Designing data governance. Commun. ACM 53, 148-152 (2010)

6. Abraham, R., Schneider, J., vom Brocke, J.: Data governance: A conceptual framework, structured review, and research agenda. International Journal of Information Management 49, 424-438 (2019)

7. Tiwana, A., Konsynski, B., Venkatraman, N.: Special Issue: Information Technology and Organizational Governance: The IT Governance Cube. Journal of Management Information Systems 30, 7-12 (2013)

8. Johannsen, W., Goeken, M.: IT-Governance - neue Aufgaben des IT-Managements. HMD - Praxis Wirtschaftsinform 250 (2006)

9. Korhonen, J., Melleri, I., Hiekkanen, K., Helenius, M.: Designing Data Governance: An Organizational Perspective. The GSTF Journal of Computing 2 (2012)

10. Otto, B., Weber, K.: Data Governance. In: Hildebrand, K., Gebauer, M., Hinrichs, H., Mielke, M. (eds.) Daten- und Informationsqualität. Auf dem Weg zur Information Excellence, pp. 269-286. Springer Vieweg, Wiesbaden (2018)

11. Thammaboosadee, S., Dumthanasarn, N.: Proposed Amendments of Public Information Act Towards Data Governance Framework for Open Government Data: Context of Thailand. In: 2018 3rd Technology Innovation Management and Engineering Science International Conference (TIMES-iCON), pp. 1-5 (2018)

12. Sharma, D., Stone, M., Ekinci, Y.: IT governance and project management: A qualitative study. J Database Mark Cust Strategy Manag 16, 29-50 (2009) 
13. Weill, P., Ross, J.W.: IT governance. How top performers manage IT decision rights for superior results. Harvard Business School Press, Boston, Mass. (2004)

14. van Grembergen, W., Haes, S. de, Guldentops, E.: Structures, Processes and Relational Mechanisms for IT Governance. In: van Grembergen, W. (ed.) Strategies for information technology governance. IGI Global (701 E. Chocolate Avenue Hershey Pennsylvania 17033 USA), Hershey, Pa (2004)

15. Smallwood, R.F.: Information Governance: Concepts, Strategies, and Best Practices (2014)

16. Kooper, M.N., Maes, R., Lindgreen, E.E.O.R.: On the governance of information: Introducing a new concept of governance to support the management of information. International Journal of Information Management 31, 195-200 (2011)

17. de Oliveira, C., Behr, A., Antônio Carlos Gastaud Maçada: Establishing the Main Mechanisms for the Accounting Information Governance: a Delphi study with Accountants. AMCIS 2019 Proceedings (2019)

18. Wrobel, A., Komnata, K., Rudek, K.: IBM data governance solutions. In: Demazeau, Y., Gao, J., Xu, G. (eds.) 2017 International Conference on Behavioral, Economic Advance in Behavioral, Economic, Sociocultural Computing (BESC). Proceedings of 4th International Conference on Behavioral, Economic, and Socio-Cultural Computing (BESC 2017), Krakow, Poland, 16-18 October 2017, pp. 1-3. IEEE, Piscataway, NJ (2017)

19. Fettke, P.: State-of-the-Art des State-of-the-Art. Wirtsch. Inform. 48, 257-266 (2006)

20. Webster, J., Watson, R.T.: Analyzing the Past to Prepare for the Future: Writing a Literature Review. MIS Quarterly 26 (2002)

21. Boisot, M., Canals, A.: Data, information and knowledge: have we got it right? Journal of Evolutionary Economics 14, 43-67 (2004)

22. Wang, R.Y.: A product perspective on total data quality management. Commun. ACM 41, 58-65 (1998)

23. Wende, K.: A Model for Data Governance - Organising Accountabilities for Data Quality Management. ACIS 2007 Proceedings (2007)

24. Weber, K.: Data Governance-Referenzmodell - Organisatorische Gestaltung des unternehmensweiten Datenqualitätsmanagements (2009)

25. Cheong, L., Chang, V.: The Need for Data Governance: A Case Study. ACIS 2007 Proceedings (2007)

26. Fürber, C., Sobota, J.: Eine Datenqualitätsstrategie für große Organisationen am Beispiel der Bundeswehr. HMD 48, 36-45 (2011)

27. Yulfitri, A.: Modeling operational model of data governance in government: Case study: Government agency X in Jakarta. In: 2016 International Conference on Information Technology Systems and Innovation (ICITSI), pp. 1-5 (2016)

28. Harrach, H.: Datenqualitätsmanagement. In: Harrach, H. (ed.) Risiko-Assessments für Datenqualität. Konzept und Realisierung, pp. 41-54. Vieweg+Teubner Verlag / GWV Fachverlage GmbH Wiesbaden, Wiesbaden (2010)

29. Pierce, E., Dismute, W.S., Yonke, C.L.: The State of Information and Data Governance. Understanding How Organizations Govern Their Information and Data Assets. IAIDQ and UALR-IQ (2008)

30. Martijn, N., Hulstijn, J., Bruijne, M. de, Tan, Y.-H.: Determining the Effects of Data Governance on the Performance and Compliance of Enterprises in the Logistics and Retail Sector. In: Janssen, M., Mäntymäki, M., Hidders, J., Klievink, B., Lamersdorf, W., van Loenen, B., Zuiderwijk, A. (eds.) Open and big data management and innovation. 14th IFIP WG 6.11 Conference on e-Business, e-Services, and e-Society, I3E 2015, Delft, The Netherlands, October 13-15, 2015 : proceedings, pp. 454-466. Springer, Cham, Heidelberg, New York, Dordrecht, London (2015) 
31. Weber, K., Otto, B., Österle, H.: Data Governance: Organisationskonzept für das konzernweite Datenqualitätsmanagement. In:, pp. 589-598 (2009)

32. Al-Ruithe, M., Benkhelifa, E., Hameed, K.: Data Governance Taxonomy: Cloud versus Non-Cloud. Sustainability 10, 95 (2018)

33. Kamioka, T., Luo, X., Tapanainen, T.: AN EMPIRICAL INVESTIGATION OF DATA GOVERNANCE: THE ROLE OF ACCOUNTABILITIES. PACIS 2016 Proceedings (2016)

34. Poor, M.: Applying Aspects of Data Governance from the Private Sector to Public Higher Education (2011)

35. Otto, B.: A morphology of the organisation of data governance. 19th European Conference on Information Systems, ECIS 2011 (2011)

36. Wang, R.Y., Strong, D.M.: Beyond Accuracy: What Data Quality Means to Data Consumers. Journal of Management Information Systems 12, 5-33 (1996)

37. Viscusi, G., Batini, C., Mecella, M.: Information Systems for eGovernment. A Quality-ofService Perspective. Springer-Verlag Berlin Heidelberg, Berlin, Heidelberg (2010)

38. Weber, K., Otto, B., Österle, H.: One Size Does Not Fit All---A Contingency Approach to Data Governance. J. Data and Information Quality 1, 1-27 (2009)

39. Tepandi, J., Lauk, M., Linros, J., Raspel, P., Piho, G., Pappel, I., Draheim, D.: The Data Quality Framework for the Estonian Public Sector and Its Evaluation. In: Hameurlain, A., Küng, J., Wagner, R., Sakr, S., Razzak, I., Riyad, A. (eds.) Transactions on Large-Scale Data- and Knowledge-Centered Systems XXXV, 10680, pp. 1-26. Springer Berlin Heidelberg, Berlin, Heidelberg (2017)

40. Chao, L.: Cloud computing for teaching and learning. Strategies for design and implementation. IGI Global (701 E. Chocolate Avenue Hershey Pennsylvania 17033 USA), Hershey, Pa (2012)

41. Begg, C., Caira, T.: Exploring the SME quandary: data governance in practise in the small to medium-sized enterprise sector. EJISE 15, 3-13 (2012)

42. Niemi, E.: Working Paper: Designing a Data Governance Framework (2011)

43. Al-Ruithe, M., Benkhelifa, E., Hameed, K.: Key Dimensions for Cloud Data Governance. In: Younas, M., Awan, I., Seah, W. (eds.) 2016 IEEE 4th International Conference on Future Internet of Things and Cloud. FiCloud 2016 : 22-24 August 2016, Vienna, Austria : proceedings, pp. 379-386. IEEE, Piscataway, NJ (2016)

44. Al-Ruithe, M., Benkhelifa, E., Hameed, K.: A Conceptual Framework for Designing Data Governance for Cloud Computing. Procedia Computer Science 94, 160-167 (2016)

45. Faria, F., Maçada, A.C., Kumar, K.: Modelo Estrutural De Governança Da Informação Para Bancos. Rev. adm. empres. 57, 79-95 (2017)

46. Lajara, T.T., Maçada, A.C.: Information governance framework: The defense manufacturing case study. 19th Americas Conference on Information Systems, AMCIS 2013 Hyperconnected World: Anything, Anywhere, Anytime 3, 1984-1993 (2013)

47. Tallon, P.P., Ramirez, R.V., Short, J.E.: The Information Artifact in IT Governance: Toward a Theory of Information Governance. Journal of Management Information Systems 30, 141$178(2013)$

48. Fleckenstein, M., Fellows, L.: Records Management. In: Fleckenstein, M., Fellows, L. (eds.) Modern data strategy, pp. 195-207. Springer, Cham (2018) 